\title{
Characterization of the Equilibrium Strategy of Fuzzy Bimatrix Games Based on $L-R$ Fuzzy Variables
}

\author{
Cun-lin Li \\ School of Management, Beifang University of Nationalities, Yinchuan 750021, China \\ Correspondence should be addressed to Cun-lin Li, bitlcl@gmail.com
}

Received 25 February 2012; Accepted 30 March 2012

Academic Editor: Yonghong Yao

Copyright (C) 2012 Cun-lin Li. This is an open access article distributed under the Creative Commons Attribution License, which permits unrestricted use, distribution, and reproduction in any medium, provided the original work is properly cited.

This paper deals with bimatrix games in uncertainty environment based on several types of ordering, which Maeda proposed. But Maeda's models was just made based on symmetrical triangle fuzzy variable. In this paper, we generalized Maeda's model to the non-symmetrical environment. In other words, we investigated the fuzzy bimatrix games based on nonsymmetrical $L-R$ fuzzy variables. Then the pseudoinverse of a nonconstant monotone function was given and the concept of crisp parametric bimatrix games was introduced. At last, the existence condition of Nash equilibrium strategies of the fuzzy bimatrix games is proposed and (weak) Pareto equilibrium of the fuzzy bimatrix games was obtained through the Nash equilibrium of the crisp parametric bimatrix.

\section{Introduction}

Nash presented noncooperative game theory $[1,2]$ based on the assumption that each player has a well-defined quantitative utility function over a set of the player's strategy, each player attempts to optimize his own expected payoffs, and each is assumed to know the extensive game completely. In this paper, above assumption had been violated involving complex problems in economics, engineering, social and political sciences due to the difficulty inherent in defining an adequate payoff function for each player in these types of problems. In other words, while modeling the noncooperative games, each player can not give the exact payoffs in practice because of the complexity and uncertainty of the problems. So the gain or payoff function is not always evaluated by a crisp number. It should be formulated semantically, in such terms as excellent, good, sufficiently reliable, durable, or resistant. Fortunately, in this paper, we have another utility to measure the uncertainty: the fuzzy set theory. Indeed, a 
fuzzy variable is used to present the payoffs of the players. For example, one player's payoff is about 100 thousand dollars. Since the expected payoffs of the player are fuzzy variable, we should define new concepts of equilibrium strategy to investigate their properties.

Butnariu [3] started with the work of noncooperative fuzzy games, he proposed a game to be fuzzy when the players have fuzzy preferences. Campos [4] modeled the twoperson zero-sum games with fuzzy payoffs by fuzzy programming and transformed the fuzzy programming into a linear programming problems through Yager's fuzzy variable ordering method [5]. Nishizaki and Sakawa [6,7] investigated single- and multiobjective games and presented max-min algorithm with respect to a degree of attainment of the aggregated fuzzy goal. Through maximizing the minimum of the fuzzy expected payoff and fuzzy goal, they transform their model into a fractional programming problem and compute the fractional programming by a relaxed method., Bector et al. [8, 9] and Vijay e al. $[10,11]$ modeled the noncooperative games in uncertainty by fuzzy programming problem and computed their model by the fuzzy dual programming. Takashi $[12,13]$ presented three kinds of equilibrium strategies of fuzzy matrix games based on especial symmetric triangular fuzzy variable and investigated the existence condition of these equilibrium strategies. But it is partial acceptance to modeling payoffs by symmetric triangular fuzzy variable.

This paper was going to generalize Maeda's model and investigate all types of equilibrium strategies based on more general asymmetric $L-R$ fuzzy variables. Then we introduce the crisp parametric matrix games and its equilibrium strategies in order to find the Pareto equilibrium strategies of fuzzy bimatrix games. For that purpose, this paper is organized as follows. In Section 2, we introduce some basic definitions and notations about fuzzy set theory. Then, several fuzzy ordering that Ramík [14] and Maeda presented were introduced. And the pseudoinverse of a nonconstant monotone function was given. In Section 3, we focus on the condition of different equilibrium strategies. Especially, the relation between the Nash equilibrium strategies of crisp bimatrix parametric games and Pareto equilibrium strategies of fuzzy bimatrix games is established.

\section{Preliminaries}

Definition 2.1. A $L-R$ fuzzy variable $\tilde{a}$ is a fuzzy set defined on the space of real number, whose membership functions $\mu_{\tilde{a}}: \mathcal{R} \rightarrow[0,1]$ as following

$$
\mu_{\tilde{a}}(x)= \begin{cases}L\left(\frac{a-x}{l}\right), & x \leq a, l \geq 0, \\ R\left(\frac{x-a}{r}\right), & x \geq a, r \geq 0,\end{cases}
$$

where, $L, R: R \rightarrow[0,1]$ are not constant and left continuous function, they satisfy the followings: (1) $L(x)=L(-x), R(x)=R(-x)$; (2) $L(0)=R(0)=1, L(1)=R(1)=0$; (3) they are nonincreasing on $[0,+\infty)$. The $L-R$ fuzzy variable is denoted by $\tilde{a}=(a, l, r)_{L-R}, a, l$ and $r$ are called the center, left extension, and right extension, respectively.

Remark 2.2. Notice that the domains of $L(t)$ and $R(t)$, which are defined in Definition 2.1, are $[0,+\infty)$, respectively. Moreover, for $t \in[1,+\infty), L(t)=R(t)=0$ holds.

We denote the $L-R$ fuzzy variable set as $\mathcal{F}$, for $\tilde{a} \in \mathcal{F}, \alpha \in[0,1], \tilde{a}_{\alpha} \triangleq\left\{x \mid \mu_{\tilde{a}}(x) \geq\right.$ $\alpha, x \in R\}$ is called as $\alpha$-level set of $\tilde{a}$. $\tilde{a}_{0} \triangleq\left\{x \mid \mu_{\tilde{a}}(x)>0, x \in \mathcal{R}\right\}$ is called support of $\tilde{a}$. In the following of the paper, we denote $a_{\alpha}^{R} \triangleq \sup \tilde{a}_{\alpha}, a_{\alpha}^{L} \triangleq \inf \tilde{a}_{\alpha}$, and $\tilde{a}_{\alpha}=\left[a_{\alpha}^{L}, a_{\alpha}^{R}\right]$. 
Definition 2.3. Let $f:[a, b] \rightarrow[c, d]$ be a monotone function, where $[a, b]$ and $[c, d]$ are closed subintervals of the extended real line $[-\infty,+\infty]$. The pseudoinverse $f^{(-1)}(y):[c, d] \rightarrow$ $[a, b]$ of $f$ is defined by

$$
f^{(-1)}(y)= \begin{cases}\sup \{x \in[a, b] \mid f(x)<y\}, & \text { if } f(a)<f(b), \\ \sup \{x \in[a, b] \mid f(x) \geq y\}, & \text { if } f(a)>f(b), \\ a, & \text { if } f(a)=f(b) .\end{cases}
$$

Example 2.4. Let $R(t)$ be a function defined as Definition 2.1, the graph of $R(t)$ is given in Figure 1. The graph of the pseudoinverse $R^{(-1)}(t)$ is given in Figure 2. These pictures also indicate how to construct the pseudoinverse of a non-constant monotone function.

Remark 2.5. According to Remark 2.2 and Definition 2.3, the domains of $L^{(-1)}(t)$ and $R^{(-1)}(t)$ are $[0,1]$.

Next, we introduce the extension principle that Zadeh proposed in [15], which has become an important tool in fuzzy theory and its applications.

Definition 2.6. (i) Let $X, Y$ be two crisp set, $\mathscr{F}(X)$ and $\mathscr{F}(Y)$ are two fuzzy variable set defined on $X$ and $Y$. The function $f: X \rightarrow Y$ induces another function $\tilde{f}: \mathcal{F}(X) \rightarrow \mathcal{F}(Y)$ defined on each fuzzy set $\tilde{u}$ on $X$ by

$$
\tilde{f}(\tilde{u})(y)=\sup _{x \in X, f(x)=y} u(x)
$$

Let $X_{i}, i=1,2, \ldots, n, Y$ be crisp set, $\mathscr{F}\left(\prod_{i=1}^{n} X_{i}\right)$ and $\mathscr{F}(Y)$ are two fuzzy variable set defined on $\prod_{i=1}^{n} X_{i}$ and $Y$. The function $f: X \rightarrow Y$ induces another function $\tilde{f}: \mathcal{F}\left(\prod_{i=1}^{n} X_{i}\right) \rightarrow \mathcal{F}(Y)$ defined on each fuzzy set on $\prod_{i=1}^{n} X_{i}$ by

$$
\tilde{f}\left(\tilde{u}_{1}, \tilde{u}_{2}, \ldots, \tilde{u}_{n}\right)(y)=\sup _{f\left(x_{1}, x_{2}, \ldots, x_{n}\right)=y} \min _{i} u_{i}\left(x_{i}\right) .
$$

Based on the extension principle, we introduce the following definitions.

Definition 2.7. Let $\tilde{a}, \tilde{b} \in \mathcal{F}$ be $L-R$ fuzzy variables, $c \in \mathcal{R}$, then, the membership function of the sum of two fuzzy variables $\tilde{a}$ and $\tilde{b}$, the scalar product of $\tilde{a}$ and $c$ are defined as following:

(i) $\mu_{\tilde{a}+\tilde{b}}(x)=\sup _{x=\mu+v} \min \left\{\mu_{\tilde{a}}, \mu_{\tilde{b}}\right\}$,

(ii) $\mu_{c \tilde{a}}(x)=\max \left\{\sup _{x=c \mu} \mu \tilde{a}, 0\right\}$, with $\sup \{\phi\}=-\alpha$.

Lemma 2.8. Let $\tilde{a}=(a, l, r)_{L-R}, \tilde{b}=(b, m, n)_{L-R} \in \mathcal{F}$ be L-R fuzzy variables, $c \in \mathcal{R}^{+}$, it holds that

(i) $c \tilde{a}=(c a, c l, c r)_{L-R}$

(ii) $\tilde{a}+\tilde{b}=(a+b, l+m, r+n)_{L-R}$.

Definition 2.9. Let $\mathcal{R}^{n}$ be $n$-dimensional Euclidean space, $x=\left(x_{1}, x_{2}, \ldots, x_{n}\right) \in \mathcal{R}^{n}, x_{i} \in \mathcal{R}$ $(i=1,2, \ldots, n)$. 


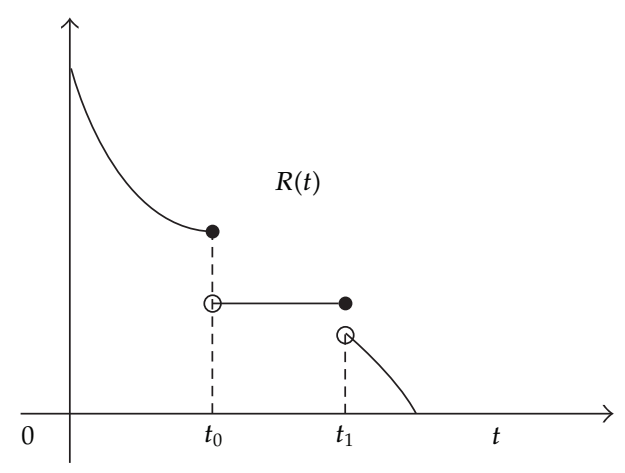

Figure 1: Monotone function $R(t)$.

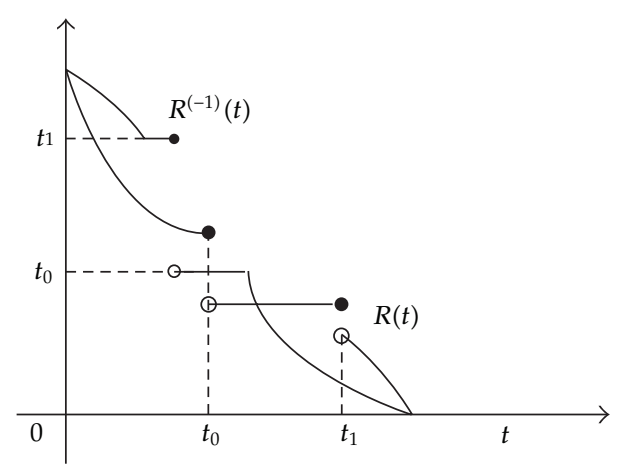

Figure 2: $R(t)$ and its pseudoinverse.

(i) $x \leqq y$ if and only if $x_{i} \leqslant y_{i}$ holds,

(ii) $x \leq y$ if and only if $x \leqq y$ and $x \neq y$ holds,

(iii) $x<y$ if and only if $x_{i}<y_{i}$ holds.

Definition 2.10 (see [14]). Let $\tilde{a}, \tilde{b} \in \mathcal{F}$ be $L-R$ fuzzy variables, then,

(i) $\tilde{b}$ dominates $\tilde{a}$ (denote by $\tilde{a} \precsim \tilde{b})$ if and only if $\left(a_{\alpha}^{L}, a_{\alpha}^{R}\right) \leqq\left(b_{\alpha}^{L}, b_{\alpha}^{R}\right)$ holds with $\alpha \in$ $[0,1]$,

(ii) $\tilde{b}$ strictly dominates $\tilde{a}$ (denote by $\tilde{a} \preccurlyeq \tilde{b})$ if and only if $\tilde{a} \precsim \tilde{b}$ and $\left(a_{\alpha}^{L}, a_{\alpha}^{R}\right) \leq\left(b_{\alpha}^{L}, b_{\alpha}^{R}\right)$ holds with $\alpha \in[0,1)$,

(iii) $\tilde{b}$ strongly dominates $\tilde{a}$ (denote by $\tilde{a}<\tilde{b})$ if and only if $\left(a_{\alpha}^{L}, a_{\alpha}^{R}\right)<\left(b_{\alpha}^{L}, b_{\alpha}^{R}\right)$ holds with $\alpha \in[0,1]$,

(iv) $\tilde{b}$ is equal to $\tilde{a}$ (denote by $\tilde{a}=\tilde{b})$ if and only if $\left(a_{\alpha}^{L}, a_{\alpha}^{R}\right)=\left(b_{\alpha}^{L}, b_{\alpha}^{R}\right)$ holds with $\alpha \in$ $[0,1]$.

Theorem 2.11. Let $\tilde{a}=(a, l, r)_{L-R}, \tilde{b}=(b, m, n)_{L-R} \in \mathcal{F}$ be L-R fuzzy variables,

(i) $\tilde{a} \precsim \tilde{b}$ if and only if $\max \{m-l, 0\} \leqslant b-a$ and $\max \{r-n, 0\} \leqslant b-a$ hold,

(ii) $\tilde{a}<\tilde{b}$ if and only if $\max \{m-l, 0\}<b-a$ and $\max \{r-n, 0\}<b-a$ hold. 
Proof. Just prove (i), the proof of (ii) is similar to (i). Let

$$
\mu_{\tilde{a}}(x)=\left\{\begin{array}{ll}
L\left(\frac{a-x}{l}\right), & x \leq a, l \geq 0, \\
R\left(\frac{x-a}{r}\right), & x \geq a, r \geq 0,
\end{array} \quad \mu_{\tilde{b}}(x)= \begin{cases}L\left(\frac{b-x}{m}\right), & x \leq b, m \geq 0, \\
R\left(\frac{x-b}{n}\right), & x \geq b, n \geq 0\end{cases}\right.
$$

be the membership function of fuzzy variables $\tilde{a}$ and $\tilde{b}$ (see Figure 3), respectively.

From Definition 2.1, for $\alpha \in[0,1]$, it holds that

$$
\alpha=L\left(\frac{a-x}{l}\right)=L\left(\frac{b-y}{m}\right)=R\left(\frac{x-a}{r}\right)=R\left(\frac{x-b}{n}\right) .
$$

By $L(0)=R(0)=1$, setting $\alpha=1$ it holds that $a_{1}^{L}=a, b_{1}^{R}=b$. Combining these with Definition 2.10, it implies that

$$
a \leq b .
$$

By $L(1)=R(1)=0$, setting $\alpha=0$, it holds that $a_{0}^{L}=a-l, a_{0}^{R}=a+r ; b_{0}^{L}=b-m, b_{0}^{R}=b+n$. From Definition 2.10, it implies

$$
a_{0}^{L}=a-l \leq b_{0}^{L}=b-m, \quad a_{0}^{R}=a+r \leq b_{0}^{R}=b+n .
$$

Combining (2.7) and (2.8), we have

$$
\begin{aligned}
& \max \{m-l, 0\} \leqslant b-a, \\
& \max \{r-n, 0\} \leqslant b-a .
\end{aligned}
$$

Conversely, for any $\alpha \in[0,1]$, it holds (2.6). Using the pseudoinverse of $L$ and $R$, we obtain

$$
\begin{aligned}
& \tilde{a}_{\alpha}=\left[a_{\alpha}^{L}, a_{\alpha}^{R}\right]=\left[a-l L^{(-1)}(\alpha), a+r R^{(-1)}(\alpha)\right], \\
& \tilde{b}_{\alpha}=\left[b_{\alpha}^{L}, b_{\alpha}^{R}\right]=\left[b-m L^{(-1)}(\alpha), b+n R^{(-1)}(\alpha)\right] .
\end{aligned}
$$

Then

$$
\begin{aligned}
& b_{\alpha}^{L}-a_{\alpha}^{L}=b-m L^{(-1)}(\alpha)-a+l L^{(-1)}(\alpha)=(b-a)-(m-l) L^{(-1)}(\alpha), \\
& b_{\alpha}^{R}-a_{\alpha}^{R}=b+n R^{(-1)}(\alpha)-a-r R^{(-1)}(\alpha)=(b-a)-(r-n) R^{(-1)}(\alpha) .
\end{aligned}
$$

From (i) of this Theorem and Remark 2.5, it obviously that $a_{\alpha}^{L} \leq b_{\alpha}^{L}$ and $a_{\alpha}^{R} \leq b_{\alpha}^{R}$, in other words, $\tilde{a} \precsim \tilde{b}$. 


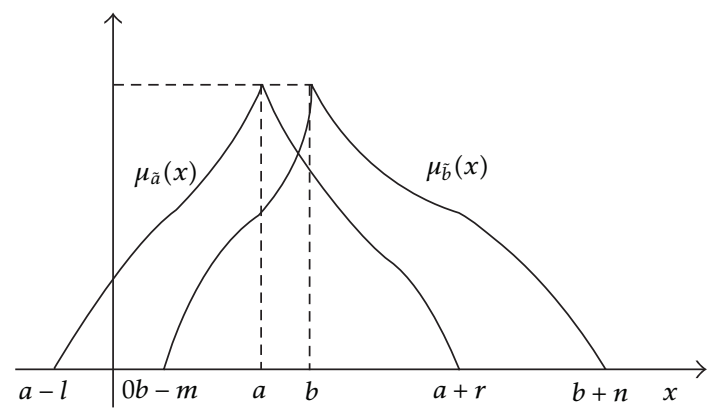

Figure 3: the membership function of fuzzy variables $\tilde{a}$ and $\tilde{b}$.

Lemma 2.12 (see [16]). Let $f\left(x_{1}, x_{2}, \ldots, x_{n}\right)$ be any usual continuous function with respect to $\left(x_{1}\right.$, $\left.x_{2}, \ldots, x_{n}\right), \tilde{x}_{i}, i=1,2, \ldots, n$, be fuzzy variables, and $\tilde{x}_{i \alpha}=\left[x_{\alpha i}^{L}, x_{\alpha i}^{L}\right], i=1,2, \ldots, n$, be corresponding $\alpha$-level sets, respectively. Then, for the $\alpha$-level set of the function $\tilde{f}\left(\tilde{x}_{1}, \tilde{x}_{2}, \ldots, \tilde{x}_{n}\right)$ with fuzzy variables, the following relation holds:

$$
\left(\tilde{f}\left(\tilde{x}_{1}, \tilde{x}_{2}, \ldots, \tilde{x}_{n}\right)\right)_{\alpha}=\left\{f\left(x_{1}, x_{2}, \ldots, x_{n}\right) \in \mathcal{R} \mid x_{i} \in\left[x_{i \alpha}^{L}, x_{i \alpha}^{R}\right], i=1,2, \ldots, n\right\} .
$$

In short, one denotes $\left\{f\left(x_{1}, x_{2}, \ldots, x_{n}\right) \in \mathcal{R} \mid x_{i} \in\left[x_{i \alpha}^{L}, x_{i \alpha}^{R}\right], i=1,2, \ldots, n\right\}$ by $f\left(\left[x_{i \alpha}^{L}, x_{i \alpha}^{R}\right]\right)$, $i=1,2, \ldots, n$.

\section{Equilibrium Strategies of Bimatrix Games with Fuzzy Payoffs}

When we apply the game theory to model some practical problems which we encounter in real world, we have to find the values of payoffs exactly. However, it is difficult to know the exact values of payoffs and we just know the values of payoffs approximately. In such situations, we should model the payoffs of the player as a fuzzy variable, so the expected payoffs of the game should be fuzzy valued. Since there are no concepts of equilibrium strategies to be accepted widely, it is an important task to define the concepts of equilibrium strategies and investigate their properties in fuzzy viroment. In this paper, the payoffs of strategy pair $(i, j) \in S_{I} \times S_{J}$ will be modeled as $L-R$ fuzzy variable, such as $\tilde{a}=(a, l, r)_{L-R}$, $\tilde{b}=(b, h, z)_{L-R} \in \mathcal{F}$.

Definition 3.1. Let $M=\{1,2, \ldots, m\}$ and $N=\{1,2, \ldots, n\}$ be sets of all pure strategies of Player $I$ and Player $J$, respectively. Their mixed strategies are probability distribution on their pure strategies. The set of mixed strategies for Player I is represented by

$$
S_{I}=\left\{\left(x_{1}, x_{2}, \ldots, x_{m}\right) \in R^{m} \mid x_{i} \geqslant 0, i=1,2, \ldots, m, \sum_{i=1}^{m} x_{i}=1\right\},
$$

where $R^{m}$ is a set of $m$-dimensional real numbers space. Similarly, the set of mixed strategies for Player $J$ is represented by

$$
S_{J}=\left\{\left(y_{1}, y_{2}, \ldots, y_{n}\right) \in \mathcal{R}^{n} \mid y_{i} \geqslant 0, i=1,2, \ldots, n, \sum_{i=1}^{n} y_{i}=1\right\} .
$$


Definition 3.2. Let Player $I$ choose a mixed strategy $x \in S_{I}$ and Player $J$ choose a mixed strategy $y \in S_{J}$. A game is said to be fuzzy bimatrix game if $\tilde{a}_{i j}$ represents the income of player $I$ and $\widetilde{b}_{i j}$ represent the incomes of player $J$, denoted by $\Gamma \equiv\left(\{I, J\}, S_{I}, S_{J}, \widetilde{A}, \widetilde{B}\right)$, where, the membership function of the payoffs will be given based on the experts' assessment. The expected value $E(x, y)=x^{T} \tilde{A} y=\sum_{i=1}^{m} \sum_{j=1}^{n} x_{i} \tilde{a}_{i j} y_{j}$ and $E(x, y)=x^{T} \widetilde{B} y=\sum_{i=1}^{m} \sum_{j=1}^{n} x_{i} \tilde{b}_{i j} y_{j}$ are called the expected of players. The matrix:

$$
\tilde{A}=\left(\begin{array}{ccc}
\tilde{a}_{11} & \cdots & \tilde{a}_{1 n} \\
\vdots & \ddots & \vdots \\
\tilde{a}_{m 1} & \cdots & \tilde{a}_{m n}
\end{array}\right), \quad \widetilde{B}=\left(\begin{array}{ccc}
\tilde{b}_{11} & \cdots & \tilde{b}_{1 n} \\
\vdots & \ddots & \vdots \\
\tilde{b}_{m 1} & \cdots & \tilde{b}_{m n}
\end{array}\right)
$$

is payoff matrix of Player $I$ and Player $J$, respectively.

In the following of this paper, we denote $A=\left(a_{i j}\right)_{m \times n^{\prime}} L=\left(l_{i j}\right)_{m \times n^{\prime}} R=\left(r_{i j}\right)_{m \times n^{\prime}}$ $A_{0}^{L}=A-L, A_{0}^{R}=A+R, B=\left(b_{i j}\right)_{m \times n^{\prime}} H=\left(h_{i j}\right)_{m \times n^{\prime}} Z=\left(z_{i j}\right)_{m \times n^{\prime}} B_{0}^{L}=B-H$ and $B_{0}^{R}=B+Z$.

Definition 3.3 (see [12]). A pair $\left(x^{*}, y^{*}\right) \in S_{I} \times S_{J}$ is called Nash equilibrium strategy of fuzzy bimatrix game $\widetilde{\Gamma}$, if it holds that

(i) $x^{T} \tilde{A} y^{*} \precsim x^{* T} \tilde{A} y^{*}, x \in S_{I}$,

(ii) $x^{* T} \widetilde{B} y \precsim x^{* T} \widetilde{B} y^{*}, y \in S_{J}$.

Definition 3.4 (see [12]). A pair $\left(x^{*}, y^{*}\right) \in S_{I} \times S_{J}$ is called Pareto equilibrium strategy of fuzzy bimatrix game $\widetilde{\Gamma}$, if it holds that

(i) there exists no $x \in S_{I}$ such that $x^{* T} \widetilde{A} y^{*} \preccurlyeq x^{T} \widetilde{A} y^{*}$,

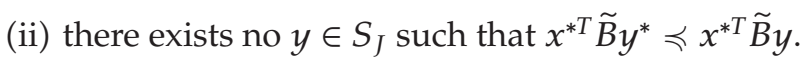

Definition 3.5 (see [12]). A pair $\left(x^{*}, y^{*}\right) \in S_{I} \times S_{J}$ is called weak Pareto equilibrium strategy of fuzzy bimatrix game $\widetilde{\Gamma}$, if it holds that

(i) there exists no $x \in S_{I}$ such that $x^{* T} \tilde{A} y^{*} \prec x^{T} \widetilde{A} y^{*}$,

(ii) there exists no $y \in S_{J}$ such that $x^{* T} \widetilde{B} y^{*} \prec x^{* T} \widetilde{B} y$.

It is obvious that, if the payoffs of fuzzy bimatrix games are all crisp number, then the above three definitions coincide with the equilibrium of a crisp bimatrix games. Therefore, these definitions are natural generalization of the crisp bimatrix games.

Lemma 3.6. Let $\tilde{A}=\left(\tilde{a}_{i j}\right)_{m \times n}$ be one of fuzzy payoff matrix of fuzzy bimatrix game $\widetilde{\Gamma}$, for $x \in S_{I}$, $y \in S_{J}, \alpha \in[0,1]$, it holds that

(i) $x^{T} \tilde{A} y \in \mathcal{F}$,

(ii) $\left(x^{T} \tilde{A} y\right)_{\alpha}=\left[x^{T} A_{\alpha}^{L} y, x^{T} A_{\alpha}^{R} y\right]$. 
Proof. It is easy to obtain (i) from Lemma 2.8. Hence we just prove (ii). Since $x^{T} A y$ is a liner function with respect to $a_{i j}$, hence it is continuous and increased with respect to $a_{i j}$, from Lemma 2.12, it is obvious that

$$
\begin{aligned}
\left(x^{T}\right. & \tilde{A} y)_{\alpha} \\
& =\left(\sum_{i=1}^{m} \sum_{j=1}^{n} x_{i} \tilde{a}_{i j} y_{j}\right)_{\alpha} \\
& =\left\{\sum_{i=1}^{m} \sum_{j=1}^{n} t_{i j} \mid t_{i j} \in\left(x_{i} \tilde{a}_{i j} y_{j}\right)_{\alpha^{\prime}} i=1,2, \ldots, m, j=1,2, \ldots, n\right\} \\
= & \left\{\sum_{i=1}^{m} \sum_{j=1}^{n} x_{i} p_{i j} y_{j} \mid p_{i j} \in\left(\tilde{a}_{i j}\right)_{\alpha^{\prime}} i=1,2, \ldots, m, j=1,2, \ldots, n\right\} \\
= & \left\{\sum_{i=1}^{m} \sum_{j=1}^{n} x_{i} p_{i j} y_{j} \mid p_{i j} \in\left[a_{i j}-L^{(-1)}(\alpha) l_{i j}, a_{i j}+R^{(-1)}(\alpha) r_{i j}\right], i=1,2, \ldots, m, j=1,2, \ldots, n\right\} \\
= & {\left[\sum_{i=1}^{m} \sum_{j=1}^{n} x_{i}\left(a_{i j}-L^{(-1)}(\alpha) l_{i j}\right) y_{j}, \sum_{i=1}^{m} \sum_{j=1}^{n} x_{i}\left(a_{i j}+R^{(-1)}(\alpha) r_{i j}\right) y_{j}\right] } \\
= & {\left[x^{T} A_{\alpha}^{L} y_{,} x^{T} A_{\alpha}^{R} y\right] . }
\end{aligned}
$$

The theorem is proved.

Theorem 3.7. Let $\widetilde{\Gamma} \equiv\left(\{I, J\}, S_{I}, S_{J}, \widetilde{A}, \widetilde{B}\right)$ be a bimatrix game with fuzzy payoffs, a pair $\left(x^{*}, y^{*}\right) \in$ $S_{I} \times S_{J}$ is the Nash equilibrium strategy of game $\tilde{\Gamma}$ if and only if the following inequalities hold with every $x \in S_{I}, y \in S_{J}$

$$
\begin{aligned}
& x^{T} A y^{*} \leqslant x^{* T} A y^{*}, \quad x^{* T} B y \leqslant x^{* T} B y^{*}, \\
& x^{T} A y^{*}-x^{T} L y^{*} \leqslant x^{* T} A y^{*}-x^{* T} L y^{*}, \quad x^{* T} B y-x^{* T} H y \leqslant x^{* T} B y^{*}-x^{* T} H y^{*}, \\
& x^{T} A y^{*}+x^{T} R y^{*} \leqslant x^{* T} A y^{*}+x^{* T} R y^{*}, \quad x^{* T} B y+x^{* T} Z y \leqslant x^{* T} B y^{*}+x^{* T} Z y^{*} .
\end{aligned}
$$

Proof. Let the pair $\left(x^{*}, y^{*}\right) \in S_{I} \times S_{J}$ be Nash equilibrium of game $\widetilde{\Gamma}$. According to Theorem 2.11 and Definition 3.3, we have

$$
\begin{aligned}
& \max \left\{x^{* T} L y^{*}-x^{T} L y^{*}, 0\right\} \leqslant x^{* T} A y^{*}-x^{T} A y^{*}, \\
& \max \left\{x^{T} R y^{*}-x^{* T} R y^{*}, 0\right\} \leqslant x^{* T} A y^{*}-x^{T} A y^{*},
\end{aligned}
$$


Journal of Applied Mathematics

$$
\begin{aligned}
& \max \left\{x^{* T} H y^{*}-x^{* T} H y, 0\right\} \leqslant x^{* T} B y^{*}-x^{* T} B y, \\
& \max \left\{x^{* T} Z y-x^{* T} Z y^{*}, 0\right\} \leqslant x^{* T} B y^{*}-x^{* T} B y .
\end{aligned}
$$

From (3.8), it indicates that

$$
x^{* T} L y^{*}-x^{T} L y^{*} \leqslant x^{* T} A y^{*}-x^{T} A y^{*}, \quad 0 \leqslant x^{* T} A y^{*}-x^{T} A y^{*} .
$$

By rearranging, it holds

$$
x^{T} A y^{*}-x^{T} L y^{*} \leqslant x^{* T} A y^{*}-x^{* T} L y^{*}, \quad x^{T} A y^{*} \leqslant x^{* T} A y^{*} .
$$

In the same way, form (3.9), it holds that

$$
x^{T} R y^{*}-x^{* T} R y^{*} \leqslant x^{* T} A y^{*}-x^{T} A y^{*} .
$$

By rearranging, it holds

$$
x^{T} R y^{*}+x^{T} A y^{*} \leqslant x^{* T} A y^{*}+x^{* T} R y^{*} .
$$

By a similar way, from form (3.10) and (3.11), we have

$$
\begin{gathered}
x^{* T} B y-x^{* T} H y \leqslant x^{* T} B y^{*}-x^{* T} H y^{*}, \quad x^{* T} B y \leqslant x^{* T} B y^{*}, \\
x^{* T} Z y+x^{* T} B y \leqslant x^{* T} B y^{*}+x^{* T} Z y^{*} .
\end{gathered}
$$

By rearranging (3.13), (3.15), and (3.16) we get (3.5), (3.6), and (3.7).

Otherwise, according to (3.5), it holds,

$$
\begin{aligned}
& 0 \leqslant x^{* T} A y^{*}-x^{T} A y^{*}, \\
& 0 \leqslant x^{* T} B y^{*}-x^{* T} B y .
\end{aligned}
$$

From (3.6), it implies that

$$
\begin{aligned}
& x^{* T} L y^{*}-x^{T} L y^{*} \leqslant x^{* T} A y^{*}-x^{T} A y^{*}, \\
& x^{* T} H y^{*}-x^{T} H y^{*} \leqslant x^{* T} B y^{*}-x^{T} B y^{*}
\end{aligned}
$$

From (3.7), it follows that

$$
\begin{aligned}
& x^{T} R y^{*}-x^{* T} R y^{*} \leqslant x^{* T} A y^{*}-x^{T} A y^{*}, \\
& x^{T} Z y^{*}-x^{* T} Z y^{*} \leqslant x^{* T} B y^{*}-x^{T} B y^{*} .
\end{aligned}
$$


Combining (3.17), (3.19), and (3.21), it holds (3.8) and (3.9). Combining (3.18), (3.20), and (3.22), it holds (3.10) and (3.11). Therefore, we have Definition 3.3 by Theorem 2.11.

By the above result, from $x^{T} A y-x^{T} L y=x^{T} A_{0}^{L} y, x^{T} A y+x^{T} R y=x^{T} A_{0}^{R} y, x^{T} B y-$ $x^{T} H y=x^{T} B_{0}^{L} y, x^{T} B y+x^{T} Z y=x^{T} B_{0}^{R} y$ with Theorem 3.7, we conclude that a fuzzy bimatrix game is equivalent to the following three crisp matrix games $\Gamma_{l} \equiv\left(\{I, J\}, S_{I}, S_{J}, A_{0}^{L}, B_{0}^{L}\right), \Gamma_{c} \equiv$ $\left(\{I, J\}, S_{I}, S_{J}, A, B\right), \Gamma_{r} \equiv\left(\{I, J\}, S_{I}, S_{J}, A_{0}^{R}, B_{0}^{R}\right)$. Then the following holds.

Corollary 3.8. A pair $\left(x^{*}, y^{*}\right) \in S_{I} \times S_{J}$ is equilibrium strategy of bimatrix game $\widetilde{\Gamma}$ if and only if the pair is still to be the equilibrium strategy of these games $\Gamma_{l}, \Gamma_{c}$, and $\Gamma_{r}$.

Remark 3.9. From above existence conditions of equilibrium strategy, it is obvious that the equilibrium strategy of fuzzy bimatrix games still is equilibrium strategy of three crisp bimatrix games. It is difficult to satisfy these conditions at the same time, but in following cases, there exists a equilibrium strategy of the bimatrix games with fuzzy payoffs.

Case 1. The payoff matrix of the bimatrix games are as follows:

$$
a_{i j}=k_{1} l_{i j}, \quad a_{i j}=k_{2} r_{i j}, \quad b_{i j}=k_{3} h_{i j}, \quad b_{i j}=k_{4} z_{i j}, \quad i=1,2, \ldots, m, j=1,2, \ldots, n,
$$

where $k_{1}, k_{2}, k_{3}, k_{4} \in(0,1]$. In other words, $A=k_{1} L, A=k_{2} R, B=k_{3} H, B=k_{4} Z$.

Case 2. If $l_{i j}=l, r_{i j}=r, h_{i j}=h, z_{i j}=z, i=1,2, \ldots, m, j=1,2, \ldots, n$, where $l, r, h, z \in \mathcal{R}$.

Remark 3.10. In these cases, the fuzzy bimatrix game $\widetilde{\Gamma} \equiv\left(\{I, J\}, S_{I}, S_{J}, \widetilde{A}, \widetilde{B}\right)$ is equivalent to the crisp bimatrix game $\widetilde{\Gamma} \equiv\left(\{I, J\}, S_{I}, S_{J}, A, B\right)$.

Theorem 3.11. Let $\tilde{\Gamma} \equiv\left(\{I, J\}, S_{I}, S_{J}, \widetilde{A}, \widetilde{B}\right)$ be a fuzzy bimatrix game, $T_{I}(\widetilde{\Gamma})$, and $T_{J}(\widetilde{\Gamma})$ are the sets of the optimal strategy of Player I and Player J, respectively. Then, $T_{I}(\widetilde{\Gamma})$, and $T_{J}(\widetilde{\Gamma})$ are both nonempty closed convex set if they are nonempty.

Proof. We just present the proof of the closed convex set of $T_{I}(\widetilde{\Gamma})$, the proof of the closed convex set of $T_{J}(\widetilde{\Gamma})$ is similar to the proof of $T_{I}(\widetilde{\Gamma})$.

Let us assume $x^{*} \in T_{I}(\widetilde{\Gamma})$ and $y^{*} \in T_{J}(\widetilde{\Gamma})$, then the pair $\left(x^{*}, y^{*}\right)$ is a equilibrium strategy of the fuzzy bimatrix games. From Definition 3.3, it is obvious that

$$
\begin{gathered}
x^{* T} \tilde{A} y \precsim x^{* T} \tilde{A} y^{*}, \quad y \in S_{J}, \\
x^{T} \widetilde{B} y^{*} \precsim x^{* T} \widetilde{B} y^{*}, \quad x \in S_{I} .
\end{gathered}
$$

By Theorem 3.7 and Remark 3.9, the pair $\left(x^{*}, y^{*}\right)$ is also the equilibrium strategy of three crisp bimatrix games $\Gamma_{l} \equiv\left(\{I, J\}, S_{I}, S_{J}, A_{0}^{L}, B_{0}^{L}\right), \Gamma_{c} \equiv\left(\{I, J\}, S_{I}, S_{J}, A, B\right), \Gamma_{r} \equiv\left(\{I, J\}, S_{I}\right.$, $\left.S_{J}, A_{0}^{R}, B_{0}^{R}\right)$. In other words, $x^{*} \in T_{I}\left(\Gamma_{l}\right), x^{*} \in T_{I}\left(\Gamma_{c}\right), x^{*} \in T_{I}\left(\Gamma_{r}\right)$, then, $x^{*} \in T_{I}\left(\Gamma_{l}\right) \cap$ $T_{I}\left(\Gamma_{c}\right) \cap T_{I}\left(\Gamma_{r}\right)$.

On the other hand, $T_{I}\left(\Gamma_{l}\right), T_{I}\left(\Gamma_{c}\right)$, and $T_{I}\left(\Gamma_{l}\right)$ are all closed convex set. Therefore, $T_{I}(\tilde{\Gamma})$ is a closed convex set. 
Theorem 3.12. Let $\widetilde{\Gamma} \equiv\left(\{I, J\}, S_{I}, S_{J}, \widetilde{A}, \widetilde{B}\right)$ be a fuzzy bimatrix game, a pair $\left(x^{*}, y^{*}\right) \in S_{I} \times S_{J}$ is the Pareto equilibrium strategy of game $\widetilde{\Gamma}$ if and only if

(i) there exists no $x \in S_{I}$ such that

$$
x^{* T}\left(A_{0}^{L}, A_{0}^{R}\right) y^{*} \leq x^{T}\left(A_{0}^{L}, A_{0}^{R}\right) y^{*}, \quad x^{* T} A y^{*} \leq x^{T} A y^{*},
$$

(ii) there exists no $y \in S_{J}$ such that

$$
x^{* T}\left(B_{0}^{L}, B_{0}^{R}\right) y^{*} \leq x^{* T}\left(B_{0}^{L}, B_{0}^{R}\right) y, \quad x^{* T} B y^{*} \leq x^{* T} B y .
$$

Proof. Let

$$
\mu_{\tilde{a}_{i j}}(x)= \begin{cases}L\left(\frac{a_{i j}-x}{l_{i j}}\right), & x \leq a_{i j}, l_{i j} \geq 0, \\ R\left(\frac{x-a_{i j}}{r_{i j}}\right), & x \geq a_{i j}, r_{i j} \geq 0,\end{cases}
$$

be the membership function of $\tilde{a}_{i j}$, which is the payoff of Player $I$ based on pure strategy pair $(i, j)$. If the pair $\left(x^{*}, y^{*}\right) \in S_{I} \times S_{J}$ is the Pareto equilibrium of the game $\tilde{\Gamma}$. we assume there exists a strategy $\bar{x} \in S_{I}$ such that (3.25) holds, that is to say,

$$
x^{* T}(A-L, A+R) y^{*} \leq \bar{x}^{T}(A-L, A+R) y^{*}, \quad x^{* T} A y^{*} \leq \bar{x}^{T} A y^{*},
$$

It implies the following from above:

$$
x^{* T}(A-L) y^{*} \leq \bar{x}^{T}(A-L) y^{*}, \quad x^{* T}(A+R) y^{*} \leq \bar{x}^{T}(A+R) y^{*} .
$$

Furthermore, the above two inequalities do not occur simultaneously. Let $\alpha \in[0,1]$, then $L^{(-1)}(\alpha) \in[0,1]$ and $R^{(-1)}(\alpha) \in[0,1]$ based on Remark 2.5. From above inequalities, it indicates that

$$
\begin{aligned}
& \left(x^{* T}\left(\left(1-L^{(-1)}(\alpha)\right) A+L^{(-1)}(\alpha)(A-L)\right) y^{*}, x^{* T}\left(\left(1-R^{(-1)}(\alpha)\right) A+R^{(-1)}(\alpha)(A+R)\right) y^{*}\right) \\
& \leq\left(\bar{x}^{T}\left(\left(1-L^{(-1)}(\alpha)\right) A+L^{(-1)}(\alpha)(A-L)\right) y^{*}, \bar{x}^{T}\left(\left(1-R^{(-1)}(\alpha)\right) A+R^{(-1)}(\alpha)(A+R)\right) y^{*}\right) .
\end{aligned}
$$

By rearranging, it implies

$$
\begin{aligned}
& \left(x^{* T}\left(A-L^{(-1)}(\alpha) L\right) y^{*}, x^{* T}\left(A+R^{(-1)}(\alpha) R\right) y^{*}\right) \\
& \quad \leq\left(\bar{x}^{T}\left(A-L^{(-1)}(\alpha) L\right) y^{*}, \bar{x}^{T}\left(A+R^{(-1)}(\alpha) R\right) y^{*}\right)
\end{aligned}
$$

that is, $x^{* T} \tilde{A} y^{*} \preccurlyeq \bar{x}^{T} \tilde{A} y^{*}$, by the Definition 2.10. This is a contradiction. 
By the manner similar to above, there exists no $y$ such that (3.26) holds.

Otherwise, let the pair $\left(x^{*}, y^{*}\right) \in S_{I} \times S_{J}$ satisfies (3.25), (3.26). We assume that there exists a strategy $\bar{x} \in S_{I}$ such that $x^{* T} \tilde{A} y^{*} \preccurlyeq \bar{x}^{T} \widetilde{A} y^{*}$ holds. From Definition 2.10, for $\alpha \in[0,1)$, it is obvious that

$$
x^{* T}\left(A_{\alpha}^{L}, A_{\alpha}^{R}\right) y^{*} \leq \bar{x}^{T}\left(A_{\alpha}^{L}, A_{\alpha}^{R}\right) y^{*}
$$

Let $\alpha$ incline to 1 and set $\alpha=0$, respectively, it holds

$$
x^{* T}\left(A_{0}^{L}, A_{0}^{R}\right) y^{*} \leq \bar{x}^{T}\left(A_{0}^{L}, A_{0}^{R}\right) y^{*}, \quad x^{* T} A y^{*} \leq \bar{x}^{T} A y^{*}
$$

This is a contradiction.

By the similar way, we have there exists no $y \in S_{J}$ such that $x^{* T} \widetilde{B} y \preccurlyeq x^{* T} \widetilde{B} y^{*}$.

Theorem 3.13. Let $\widetilde{\Gamma} \equiv\left(\{I, J\}, S_{I}, S_{J}, \widetilde{A}, \widetilde{B}\right)$ be a fuzzy bimatrix game, a pair $\left(x^{*}, y^{*}\right) \in S_{I} \times S_{J}$ is the weak Pareto equilibrium strategy of game $\widetilde{\Gamma}$ if and only if

(i) there exists no $x \in S_{I}$ such that

$$
x^{* T}\left(A_{0}^{L}, A, A_{0}^{R}\right) y^{*}<x^{T}\left(A_{0}^{L}, A, A_{0}^{R}\right) y^{*}
$$

(ii) there exists no $y \in S_{J}$ such that

$$
x^{* T}\left(B_{0}^{L}, B, B_{0}^{R}\right) y^{*}<x^{* T}\left(B_{0}^{L}, B, B_{0}^{R}\right) y
$$

Proof. The proof of this theorem is similar to the Theorem 3.12.

In the following of this paper, we will characterize the crisp parametric matrix games. Then other types of equilibrium strategy of fuzzy bimatrix games will be investigated through the crisp parametric matrix games.

Let $\widetilde{\Gamma} \equiv\left(\{I, J\}, S_{I}, S_{J}, \widetilde{A}, \widetilde{B}\right)$ be a fuzzy bimatrix game, while Player $I$ chooses pure strategy $i$ and Player $J$ chooses pure strategy $j$, we set $(1-\lambda)\left(a_{i j}-l_{i j}\right)+\lambda\left(a_{i j}+r_{i j}\right)$ to be the payoffs of player $I$ and $-\left[(1-\mu)\left(b_{i j}-h_{i j}\right)+\mu\left(b_{i j}+z_{i j}\right)\right]$ to be the payoffs of player $J$, where $\lambda, \mu \in[0,1]$. The payoff matrixes of player $I$ and $J$ are

$$
A(\lambda)=(1-\lambda)(A-L)+\lambda(A+R), \quad B(\mu)=(1-\mu)(B-H)+\mu(B+Z) .
$$

Then, we have the crisp parametric matrix game $\Gamma(\lambda, \mu) \equiv\left(\{I, J\}, S_{I}, S_{J}, A(\lambda), B(\mu)\right)$, where $\lambda$ and $\mu$ are parameters. 
Definition 3.14. For $\lambda, \mu \in[0,1]$, the pair $\left(x^{*}, y^{*}\right) \in S_{I} \times S_{J}$ is called to be the Nash equilibrium of the crisp parametric bimatrix game $\Gamma(\lambda, \mu)$, if it holds that

(i) $x^{T} A(\lambda) y^{*} \leqslant x^{* T} A(\lambda) y^{*}, x \in S_{I}$,

(ii) $x^{* T} B(\mu) y \leqslant x^{* T} B(\mu) y^{*}, y \in S_{J}$.

Lemma 3.15. There exists at least one Nash equilibrium strategy to all crisp parametric bimatrix game $\Gamma(\lambda, \mu)$ with $\lambda, \mu \in[0,1]$.

Theorem 3.16. A pair $\left(x^{*}, y^{*}\right) \in S_{I} \times S_{J}$ is the Pareto equilibrium strategy of fuzzy bi-matrix game $\widetilde{\Gamma}$, it is necessary and sufficient that there exist $\lambda, \mu \in(0,1)$ such that $\left(x^{*}, y^{*}\right)$ is the Nash equilibrium strategy of crisp bimatrix game $\Gamma(\lambda, \mu)$.

Proof. Let $\left(x^{*}, y^{*}\right)$ be the Nash equilibrium strategy of bimatrix game $\Gamma\left(\lambda_{0}, \mu_{0}\right)$, where $\lambda_{0}, \mu_{0} \in$ $(0,1)$. For $x \in S_{I}$, from Definition 3.14(i), it holds

$$
\left(1-\lambda_{0}\right) x^{T} A_{0}^{L} y^{*}+\lambda_{0} x^{T} A_{0}^{R} y^{*} \leqslant\left(1-\lambda_{0}\right) x^{* T} A_{0}^{L} y^{*}+\lambda_{0} x^{* T} A_{0}^{R} y^{*}
$$

For $y \in S_{J}$, from Definition 3.14(ii), it holds

$$
\left(1-\mu_{0}\right) x^{* T} B_{0}^{L} y+\mu_{0} x^{* T} B_{0}^{R} y \leqslant\left(1-\mu_{0}\right) x^{* T} B_{0}^{L} y^{*}+\mu_{0} x^{* T} B_{0}^{R} y^{*}
$$

First, we assume there exists $\bar{x} \in S_{I}$ such that $x^{* T} \tilde{A} y^{*} \preccurlyeq \bar{x}^{T} \tilde{A} y^{*}$ holds. From Definition 2.10, it holds

$$
\left(x^{* T} A_{0}^{L} y^{*}, x^{* T} A_{0}^{R} y^{*}\right) \leq\left(\bar{x}^{T} A_{0}^{L} y^{*}, \bar{x}^{T} A_{0}^{R} y^{*}\right) .
$$

Because $x^{* T} A_{0}^{L} y^{*}=\bar{x}^{T} A_{0}^{L} y^{*}$ and $x^{* T} A_{0}^{R} y^{*}=\bar{x}^{T} A_{0}^{R} y^{*}$ do not occur simultaneously, we have

$$
\left(1-\lambda_{0}\right) x^{* T} A_{0}^{L} y^{*}+\lambda_{0} x^{* T} A_{0}^{R} y^{*}<\left(1-\lambda_{0}\right) \bar{x}^{T} A_{0}^{L} y^{*}+\lambda_{0} \bar{x}^{T} A_{0}^{R} y^{*}, \quad \lambda_{0} \in(0,1) .
$$

This contradicts (3.37).

By the similar way, we can conclude a contradiction to (3.38).

On the other hand, let $\left(x^{*}, y^{*}\right)$ be the Pareto equilibrium strategy of fuzzy bimatrix game $\widetilde{\Gamma}$ such that conditions (i) and (ii) of Definition 3.4 hold. It show that there is no $\bar{x} \in S_{I}$ such that

$$
\left(x^{* T} A_{0}^{L} y^{*}, x^{* T} A_{0}^{R} y^{*}\right) \leq\left(\bar{x}^{T} A_{0}^{L} y^{*}, \bar{x}^{T} A_{0}^{R} y^{*}\right)
$$

holds. Then, one of the following cases happens,

Case 1.

$$
x^{* T}(A-L) y^{*}>\bar{x}^{T}(A-L) y^{*} \quad \text { or } \quad x^{* T}(A+R) y^{*}>\bar{x}^{T}(A+R) y^{*} ;
$$


Case 2.

$$
x^{* T}(A-L) y^{*}=\bar{x}^{T}(A-L) y^{*}, \quad x^{* T}(A+R) y^{*}=\bar{x}^{T}(A+R) y^{*} .
$$

If Case 1 happens, without loss of generality, we set $x^{* T}(A-L) y^{*}>\bar{x}^{T}(A-L) y^{*}$. It is obviously that there exist $\lambda_{0} \in(0,1)$ tended to zero such that

$$
\left(1-\lambda_{0}\right) x^{* T}(A-L) y^{*}+\lambda_{0} x^{* T}(A+R) y^{*}>\left(1-\lambda_{0}\right) \bar{x}^{T}(A-L) y^{*}+\lambda_{0} \bar{x}^{T}(A+R) y^{*},
$$

If Case 2 happens, for any $\lambda \in(0,1)$

$$
(1-\lambda) x^{* T}(A-L) y^{*}+\lambda x^{* T}(A+R) y^{*} \geq(1-\lambda) \bar{x}^{T}(A-L) y^{*}+\lambda \bar{x}^{T}(A+R) y^{*} .
$$

Both Cases 1 and 2 show that there exists $\lambda_{0} \in(0,1)$ such that Definition 3.14 (i) holds. By the similar way, we have Definition 3.14 (ii).

Theorem 3.17. A pair $\left(x^{*}, y^{*}\right) \in S_{I} \times S_{J}$ is the Weak Pareto equilibrium strategy of fuzzy bimatrix game $\widetilde{\Gamma}$, it is necessary and sufficient that there exist $\lambda, \mu \in[0,1]$ such that $\left(x^{*}, y^{*}\right)$ is the Nash equilibrium strategy of crisp bimatrix game $\Gamma(\lambda, \mu)$.

Proof. The proof of this theorem is similar to the Theorem 3.16.

From Theorems 3.16, 3.17 and Lemma 3.15, it is easy to get the followings.

Theorem 3.18. Let $\widetilde{\Gamma}$ be fuzzy bimatrix games. The followings hold

(i) there exists at least one Pareto equilibrium strategy to game $\widetilde{\Gamma}$;

(ii) there exists at least one weak Pareto equilibrium strategy to game $\widetilde{\Gamma}$.

\section{Conclusion}

This paper developed a new theoretical approach to deal with the bimatrix games with fuzzy payoffs. Especially, Maeda's models were generalized from symmetric triangular variable field to asymmetric L-R fuzzy variable field. By investigating crisp parametric bimatrix games, we presented a method to figure out the Nashe quilibrium strategy and (weak) Pareto equilibrium strategy of fuzzy bimatrix games. It is also easy to find that all kinds of equilibrium strategy that we defined are nature extensions of the Nash equilibrium strategy of crisp bimatrix games.

Recommendations for future work include the development of a procedure to extend the concept of two-person fuzzy games to n-person fuzzy games. Furthermore, incorporating the concept of multiobjective decision making with the definition of fuzzy noncooperative games would allow us to establish the fuzzy multiobjective games, which is desirable for situations involving complex multiobjective decision-making problems. 


\section{Acknowledgments}

This work was supported by the National Natural Science Foundation of China (no. 71161001) and the Science Foundation of Beifang University of Nationalities (no. 72010Y041).

\section{References}

[1] J. F. Nash, Jr., "Equilibrium points in n-person games," Proceedings of the National Academy of Sciences of the United States of America, vol. 36, pp. 48-49, 1950.

[2] J. Nash, "Non-cooperative games," Annals of Mathematics, vol. 54, pp. 286-295, 1951.

[3] D. Butnariu, "Fuzzy games: a description of the concept," Fuzzy Sets and Systems, vol. 1, no. 3, pp. 181-192, 1978.

[4] L. Campos, "Fuzzy linear programming models to solve fuzzy matrix games," Fuzzy Sets and Systems, vol. 32, no. 3, pp. 275-289, 1989.

[5] R. Yager, "A procedure for ordering fuzzy subsets of the unit interval," Information Sciences, vol. 24, no. 2, pp. 143-161, 1981.

[6] I. Nishizaki and M. Sakawa, Fuzzy and Multi-Objective Games for Conflict Resolution, Physica, New York, NY, USA, 2001.

[7] I. Nishizaki and M. Sakawa, "Equilibrium solutions in multiobjective bimatrix games with fuzzy payoffs and fuzzy goals," Fuzzy Sets and Systems, vol. 111, no. 1, pp. 99-116, 2000.

[8] C. R. Bector, S. Chandra, and V. Vidyottama, "Matrix games with fuzzy goals and fuzzy linear programming duality," Fuzzy Optimization and Decision Making, vol. 3, no. 3, pp. 255-269, 2004.

[9] C. R. Bector, S. Chandra et al., Fuzzy Mathematical Programming and Fuzzy Matrix Games, Springer, Berlin, Germany, 2005.

[10] V. Vijay and C. R. Bector, "Bi-matrix games with fuzzy goals and fuzzy payoffs," Fuzzy Optimization and Decision Making, vol. 3, pp. 327-344, 2004.

[11] V. Vijay, S. Chandra, and C. R. Bector, "Matrix games with fuzzy goals and fuzzy payoffs," Omega, vol. 33, no. 5, pp. 425-429, 2005.

[12] M. Takashi, "On characterization of equilibrium strategy of two-person zero-sum games with fuzzy payoffs," Fuzzy Sets and Systems, vol. 139, no. 2, pp. 283-296, 2003.

[13] M. Takashi, "Characterization of the equilibrium strategy of the bimatrix game with fuzzy payoff," Journal of Mathematical Analysis and Applications, vol. 251, no. 2, pp. 885-896, 2000.

[14] J. Ramík and J. Ímánek, "Inequality relation between fuzzy numbers and its use in fuzzy optimization," Fuzzy Sets and Systems, vol. 16, no. 2, pp. 123-138, 1985.

[15] L. A. Zadeh, "The concept of a linguistic variable and its application to approximate reasoning," Information Sciences, vol. 8, pp. 199-249, 1975.

[16] M. Sakawa and H. Yano, "Feasibility and Pareto optimality for multiobjective nonlinear programming problems with fuzzy parameters," Fuzzy Sets and Systems, vol. 43, no. 1, pp. 1-15, 1991. 


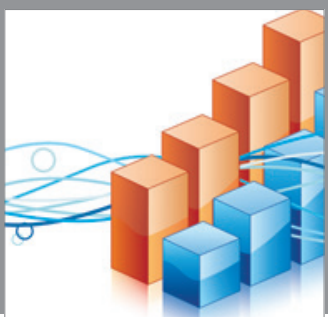

Advances in

Operations Research

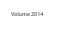

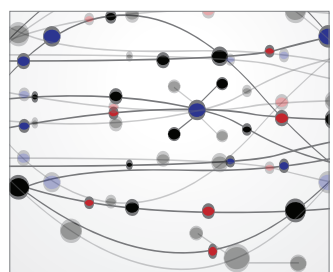

\section{The Scientific} World Journal
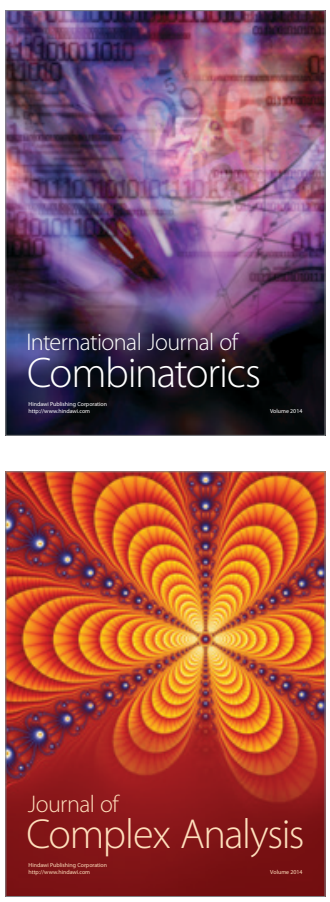

International Journal of

Mathematics and

Mathematical

Sciences
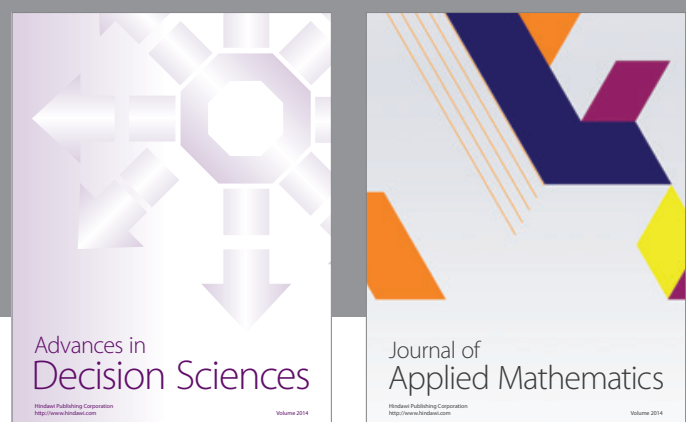

Journal of

Applied Mathematics
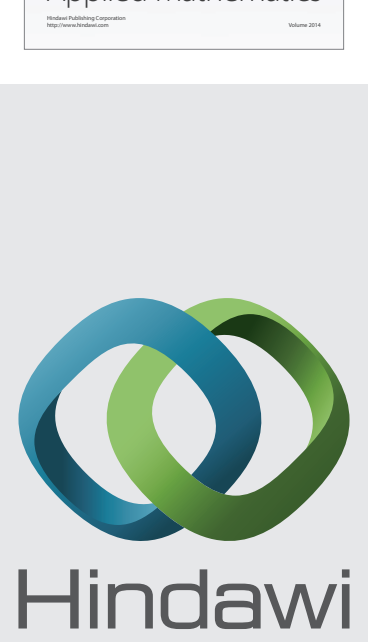

Submit your manuscripts at http://www.hindawi.com
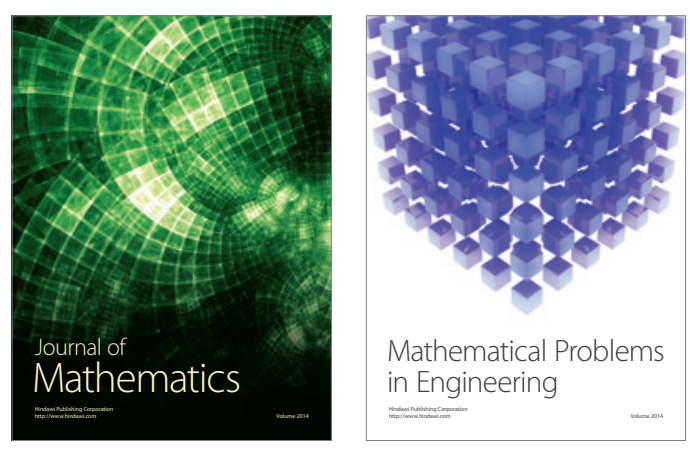

Mathematical Problems in Engineering
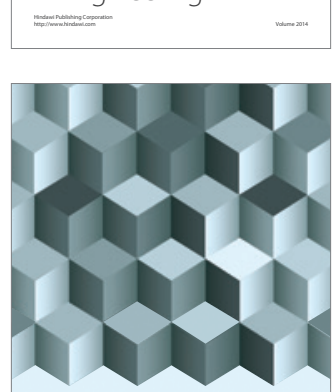

Journal of

Function Spaces
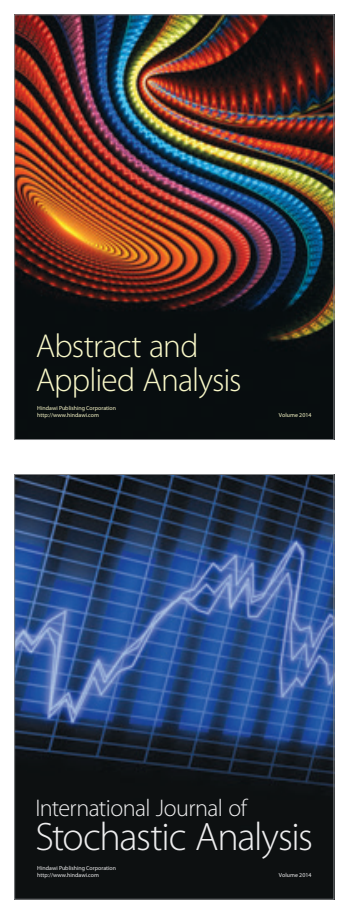

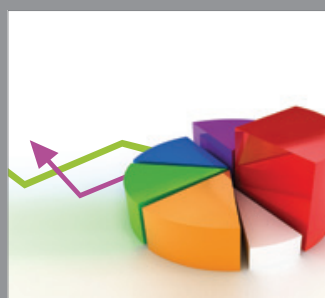

ournal of

Probability and Statistics

Promensencen
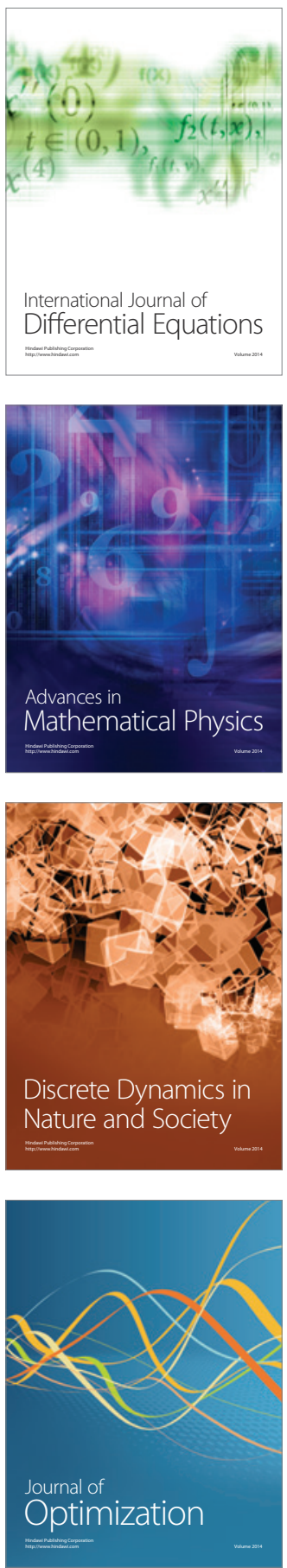